\title{
Robust Coverage under Uncertainty in Wireless Sensor Networks
}

\author{
Khuong $\mathrm{Vu}$ and Rong Zheng \\ Department of Computer Science \\ University of Houston \\ Houston, TX 77204 \\ E-mail: $\{k h u o n g . v u, r z h e n g\} @ c s . u h . e d u$
}

\section{INTRODUCTION}

Wireless sensor networks (WSNs) have been employed in monitoring the health of civil structures, tracking objects, studying the wild life habitat, among others. One critical measure of the quality of sensing (QoS) in a wireless sensor network is its coverage, i.e., "how well do the sensors observe the physical space". There has been a plural of work addressing the coverage problem in WSNs from both theoretical and system point of view [4], [3], [5], to name a few. However, most existing work assumes knowledge of the exact location of the sensors. In practice, this assumption is rarely true. Even carefully positioned in the deployment phase, sensors may be displaced due to environmental or human factors during the course of operation.

In this work, we investigate coverage properties in WSNs when the uncertainty in sensor positions is modeled by disk areas with possibly different radius centered at their nominal locations. We introduce the concept of order- $k$ max Voronoi Diagram (VD) that tessellates the interested area into regions that are closer to $k$ sensors in the worst case. An efficient polynomial algorithm is devised to construct order- $k$ max VD with complexity $O\left(k^{2}\left(\left\lceil\frac{r_{\max }}{d i s t_{\min }}\right\rceil+1\right) n \log n\right)$, where $n$ is the number of sensors, $r_{\max }$ is the maximum radius of the uncertainty disks, and dist $t_{\min }$ is the minimum distance between nominal locations of two sensors. Order- $k$ max VD is critical in determining the minimum sensing radius needed to ensure worst-case $k$-coverage, call $k$-exposure.

\section{Preliminaries}

\section{A. Network model}

Consider $n$ sensors $S=\{1,2, \ldots, n\}$ in a bounded 2$\mathrm{D}$ polygonal area region. Sensor $i$ 's location is modeled as a disk $\mathcal{C}_{i}\left(o_{i}, r_{i}\right)$ centered at its nominal location $o_{i}$ with radius $r_{i}$. Uncertainty of sensor's locations is independent. We consider binary sensing and disk coverage, where each sensor $i$ 's sensing area is modeled as a disk of radius $s_{i}$ centered at its actual location. A location $p$ is $k$-covered iff it falls in the sensing area of at least $k$ sensors.

When $r_{i}>0$, to study coverage in the worst case, we define the maximum distance from a point $p$ to a sensor $\mathcal{C}_{i}\left(o_{i}, r_{i}\right)$, as $d_{\text {max }}\left(p, \mathcal{C}_{i}\right)=\max _{q \in \mathcal{C}_{i}} d(p, q)$ where $d(\cdot, \cdot)$ is the Euclidean distance. Clearly,

$$
d_{\text {max }}\left(p, \mathcal{C}_{i}\right)=d\left(p, o_{i}\right)+r_{i} .
$$

Consequently, a location $p$ is guaranteed to be covered by sensor $i$ iff $d_{\max }\left(p, \mathcal{C}_{i}\right) \leq s_{i}$. Similarly, $p$ is guaranteed to be $k$-covered iff $\exists H \subset S,|H|=k$, such that $d_{\max }\left(p, \mathcal{C}_{i}\right) \leq s_{i}$, for all $i \in H$.

Guaranteed $k$-coverage is related to the notion of max VD and order- $k$ max VD defined as follows (the notations are borrowed from [1]).

Definition 1: For $i, j \in S$, let $D\left(\mathcal{C}_{i}, \mathcal{C}_{j}\right)=$ $\left\{x \mid d_{\max }\left(p, \mathcal{C}_{i}\right)<d_{\max }\left(p, \mathcal{C}_{j}\right)\right\}$. We define

$$
\mathcal{V}_{k}(S)=\bigcap_{i \in S, i \neq k} D\left(\mathcal{C}_{k}, \mathcal{C}_{i}\right)
$$

the maximum-Voronoi region of $\mathcal{C}_{k}$ with respect to $S$. The maximum-Voronoi diagram (max VD) of $S$ is defined as

$$
V(S)=\bigcup_{i, j \in S, i \neq j} \mathcal{V}_{i}(S) \bigcap \mathcal{V}_{j}(S)
$$

Definition 2: Let $H \subset S,|H|=k$. We define

$$
\mathcal{V}^{k}(H, S)=\bigcap_{k \in H, i \notin H} D\left(\mathcal{C}_{k}, \mathcal{C}_{i}\right)
$$

the order- $k$ maximum-Voronoi region of a set of disks $H$ with respect to $S$. The order- $k$ maximum-Voronoi diagram of $S$ is defined as

$$
V^{k}(S)=\bigcup_{H, H^{\prime} \subset S ; H \neq H^{\prime} ;|H|=\left|H^{\prime}\right|=k} \mathcal{V}^{k}(H, S) \bigcap \mathcal{V}^{k}\left(H^{\prime}, S\right)
$$

Given $\mathcal{C}_{i}\left(o_{i}, r_{i}\right), i \in S$, we are concerned with the max-min sensing radius of sensors so that a point $p$, or a polygonal area $\boldsymbol{B}$ is guaranteed to be covered. Formally,

Problem 1 (Guaranteed Point $k$-coverage): What is the minimum sensing range of the associated sensors in $S$ so that a point $p \in \boldsymbol{B}$ is guaranteed to be $k$-covered.

Problem 2 (Guaranteed Area k-coverage): What is the minimum sensing range of a set of sensors $S$ so that any point $p$ in the area $\boldsymbol{B}$ bounded by $\overline{\boldsymbol{B}}$ is $k$-covered by $S$.

We show that the sensing range for guaranteed point and area $k$-coverage can be determined from the order- $k$ max VD. The max-min sensing radius in the area $k$-coverage is also 
called $k$-exposure due to its physical meaning as the radius of largest holes in the networks (in absence of sensors). In the remaining paper, we drop the term "guaranteed" when there is no ambiguity from the context.

Note that the max-Voronoi region of a disk $\mathcal{C}_{i} \in S$ does not always exist. Consider 2 disks $\mathcal{C}_{i}\left(o_{i}, r_{i}\right)$ and $\mathcal{C}_{j}\left(o_{j}, r_{j}\right)$. Suppose $r_{i}>r_{j}$, then the max-Voronoi region of $\mathcal{C}_{i}$ does not exist if $r_{i}-r_{j}>d\left(o_{i}, o_{j}\right)$. In this case, we refer to $\mathcal{C}_{i}$ as a trivial disk. In this work, we assume that no trivial disk exists. Furthermore, for ease of presence, we consider general configurations where no point in the plane has equal maximum distance to more than 3 sensors.

\section{B. Notations}

We summarize the notations used throughout the paper as follows:

$\mathcal{C}_{k}\left(o_{k}, r_{k}\right)$ Sensor $k$ centered at $o_{k}$ with the uncertainty radius $r_{i}$. In the paper, we refer to $\mathcal{C}_{k}\left(o_{k}, r_{k}\right)$ as the disk centered at $o_{k}$ with radius $r_{k}$. We use the notion $\mathcal{C}_{k}$ for simplicity.

$S \quad$ The set of $n$ sensors $\{1, \ldots, n\}$, or equivalently, the set of $\mathrm{n}$ disks $\left\{\mathcal{C}_{1}, \mathcal{C}_{2}, \ldots, \mathcal{C}_{n}\right\}$.

$S_{i}^{\prime} \quad$ The updated $S$, in which the radius of $\mathcal{C}_{i}$ expands by a positive amount $\alpha . S_{i}^{\prime}=(S-$ $\left.\left\{\mathcal{C}_{i}\right\}\right) \bigcup\left\{\mathcal{C}_{i^{\prime}}\left(o_{i}, r_{i}+\alpha\right)\right\}$

$d_{\text {max }}\left(p, \mathcal{C}_{i}\right)$ The maximum distance from $p$ to $\mathcal{C}_{i}$. $d_{\text {max }}\left(p, \mathcal{C}_{i}\right)=d\left(p, o_{i}\right)+r_{i}$, where $d(\cdot, \cdot)$ is the Euclidean distance between two 2D points.

$\mathcal{V}_{k}(S) \quad$ The max Voronoi region corresponding to disk $\mathcal{C}_{k}$ in the max VD of $S$. We use $\mathcal{V}_{k}$ for the same purpose where no confusion occurs.

$V(S) \quad$ The max VD of the disk set $S$.

$\mathcal{V}^{k}(H, S)$ The order- $k$ max Voronoi region associated with a subset $H$ of $S$, where $|H|=k$.

$V^{k}(S) \quad$ The order- $k$ max VD of $S$. When $k=1$, $V^{k}(S) \equiv V(S)$.

$v_{i, j, k} \quad$ The max VD vertex corresponding to disks $\mathcal{C}_{i}$, $\mathcal{C}_{j}$, and $\mathcal{C}_{k}$. In figures, we use $v\left(C_{i}, C_{j}, C_{k}\right)$ instead with the same purpose for clarity.

$e_{i, j} \quad$ The edge of the max VD corresponding to disks $\mathcal{C}_{i}$ and $\mathcal{C}_{j} . e_{i, j}$ is a hyperbola segment or an infinite hyperbola. Again, we use $e\left(C_{i}, C_{j}\right)$ in figures, instead.

$\boldsymbol{B}$ The polygonal interested area to be covered.

$\bar{B} \quad$ The boundary of $\boldsymbol{B}$.

$\mathcal{F}(H) \quad$ The part of $\mathcal{V}^{k}(H, S)$ that is inside $\boldsymbol{B}$, i.e., $\mathcal{F}(H)=\boldsymbol{B} \cap \mathcal{V}^{k}(H, S)$.

$b_{i, j} \quad$ The locus of points $p$ such that $d_{\max }\left(p, \mathcal{C}_{i}\right)=$ $d_{\max }\left(p, \mathcal{C}_{j}\right) . b_{i, j}$ is a hyperbola with foci being $o_{i}$ and $o_{j}$, and possibly degenerates into straight line when $r_{i}=r_{j}$. We refer to $b_{i, j}$ as the bisector of $o_{i}$ and $o_{j}$.

\section{COnSTRUCTION OF ORDER- $k$ MAX VDs}

We provide the algorithm to construct the order- $k$ max VD for robust $k$-coverage. We start from the order-1 max VD and then devise an incremental algorithm to construct the order- $k$ max VD from the order- $(k-1)$ max VD.

\section{A. Max VD}

We start the analysis by stating that the max VD defined in Definition 1 is a tessellation of the plane without proof.

Lemma 1: If the circle $C$ expanding from a point $p$ in the plane is internally tangent to exactly one disk, $\mathcal{C}_{i}$, then $p \in \mathcal{V}_{i}$. If $C$ is internally tangent to exactly 2 disks, $\mathcal{C}_{i}$ and $\mathcal{C}_{j}$, then $p \in e_{i, j}$. If $C$ is internally tangent to 3 disks or more, $p$ is a max VD vertex corresponding to those disks.

Two disks $\mathcal{C}_{i}, \mathcal{C}_{j} \in S$ are neighbors iff they share at least an edge in $V(S)$. The basic idea of the construction of max VD is, starting from a regular VD with no uncertainty, we expand the radii of $\mathcal{C}_{i}$ 's in turn to the targeted values. Consider the max VD $V(S)$. Suppose that we expand $\mathcal{C}_{i}\left(o_{i}, r_{i}\right)$ to obtain $\mathcal{C}_{i^{\prime}}\left(o_{i}, r_{i}+\alpha\right)(\alpha>0)$ such that $\mathcal{V}_{i^{\prime}}\left(S_{i}^{\prime}\right) \neq \emptyset$, then $\mathcal{V}_{i^{\prime}}$ is obtained by shrinking $\mathcal{V}_{i}$. Meanwhile, $\mathcal{V}_{k}$, where $\mathcal{C}_{k}$ is a neighbor of $\mathcal{C}_{i}$ in $V(S)$, expands toward $o_{i}$. Now given $V\left(S^{\prime}\right), S^{\prime}=\left\{\mathcal{C}_{1^{\prime}}\left(o_{1}, r_{1}^{\prime}\right), \mathcal{C}_{2^{\prime}}\left(o_{2}, r_{2}^{\prime}\right), \ldots, \mathcal{C}_{n^{\prime}}\left(o_{n}, r_{n}^{\prime}\right)\right\}$, where $r_{i}^{\prime} \leq r_{i}, i=\{1, \ldots, n\}, V(S)$ is constructed by iteratively expanding $\mathcal{C}_{i^{\prime}}$, while fixing $\left(S^{\prime}-\left\{\mathcal{C}_{i^{\prime}}\right\}\right)$, until $r_{i}^{\prime}=r_{i}$, $i=\{1, \ldots, n\}$. As an example, consider Figure 1. The original $\max$ VD of $S=\left\{\mathcal{C}_{1}, \ldots, \mathcal{C}_{7}\right\}$ is presented in solid arcs. As $\mathcal{C}_{6}$ expands, $\mathcal{V}_{6}$ shrinks and the edges between $\mathcal{C}_{6}$ and its neighbors move toward $o_{6}$ (the dashed arcs). Eventually, $e_{1,2}$ and $e_{2,3}$ meet at point $p$, and edges of $\mathcal{C}_{6}$ are shown as the dotted arcs. With further expansion of $\mathcal{C}_{6}, e_{2,6}$ ceases to exist, and $e_{1,3}$ (presented in the figure by the edge with arrows at two ends) appears in the diagram. We refer those to edgedeath/birth, respectively. Now, $e_{3,6}$ and $e_{1,6}$ share a vertex.

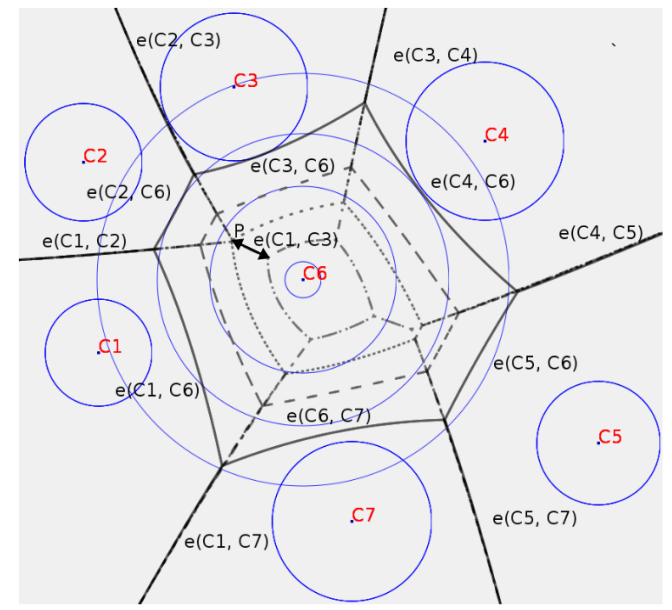

Fig. 1: Illustration of the evolution of $\mathcal{C}_{6}$ 's edges when $\mathcal{C}_{6}$ expands.

Algorithm 1 sketches the procedure of max VD construction. It takes the nominal locations of the sensors and the radii of the uncertainty disks as input. The algorithm operates by iteratively expanding the disks (line 2) from zero radii until the desired sizes are met. In the initialization phase, with zero radii, max VD is equivalent to the regular point VD (line 1). 
In each round, the algorithm selects the smallest disk yet reaching its desired size (line 3 ). Let the disk be $\mathcal{C}_{j}$. The amount of expansion is given by (line $4-$ line 7 ):

$$
\alpha=\min \left\{\min _{e_{j, k} \neq \emptyset, k \neq j}\left\{d\left(o_{k}, o_{j}\right)+r_{k}^{\prime}-r_{j}^{\prime}-\epsilon, r_{j}-r_{j}^{\prime}\right\}\right\},
$$

where $\epsilon$ is a small positive constant. (1) ensures that disk $\mathcal{C}\left(o_{j}, r_{i}^{\prime}\right)$ is not internally tangent to any other disks, thus does not create any trivial disk while expanding. Lines $8-14$ perform the disk expansion with awareness of the events of edge death/birth.

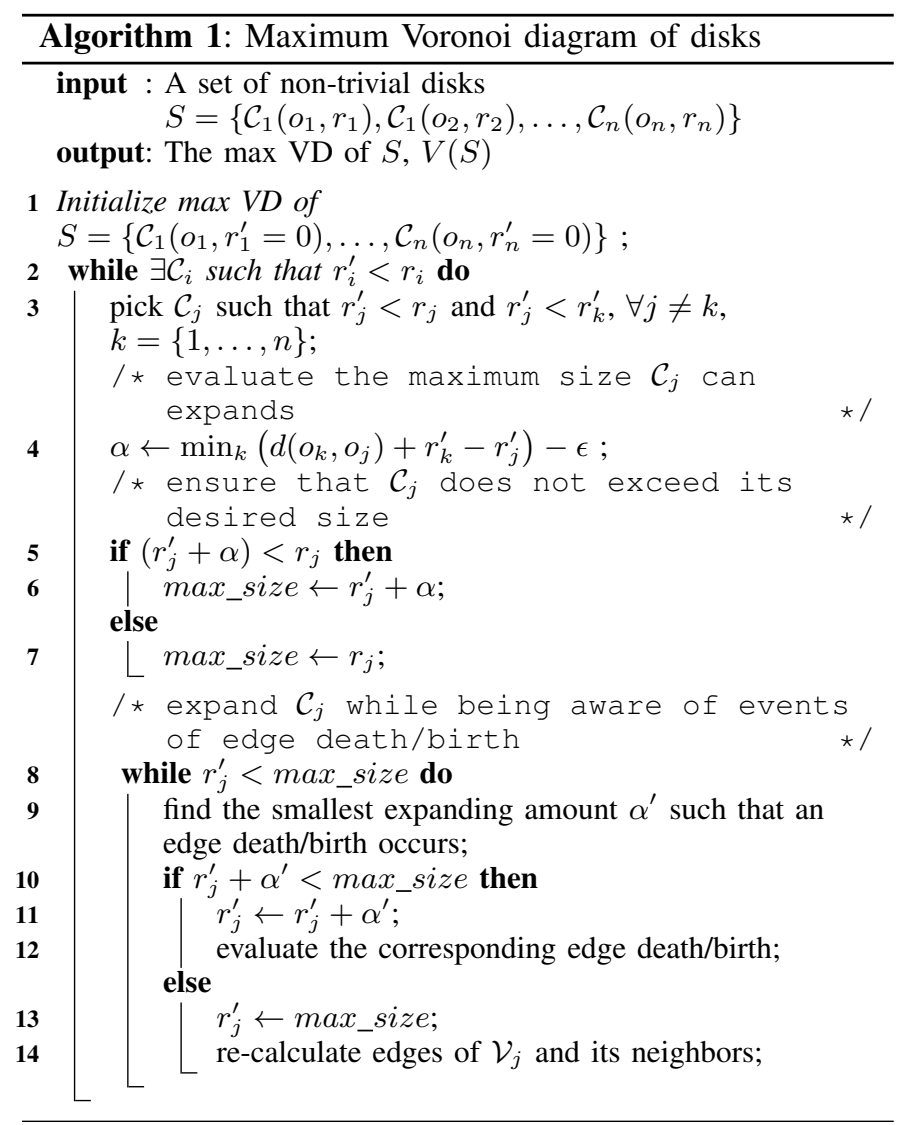

The maximum Voronoi diagram of disks can be constructed using Algorithm 1 with the computational complexity:

$$
O\left(\left(\left\lceil\frac{r_{\max }}{\text { dist }_{\min }}\right\rceil+1\right) n \log n\right)
$$

In practical deployments, the distances between the nominal locations are usually larger than the uncertainty radii. Therefore, it is reasonable to expect that the total cost is dominated by the cost of constructing the Voronoi diagram of points at the nominal locations.

\section{B. Order-k max VD}

In his seminal work[2], Lee proposed an incremental algorithm to construct order- $k$ point VDs. We extend the techniques in [2] to devise an incremental procedure for constructing order- $k$ max VDs. Like order-1 max VDs, order$k$ max VDs are characterized by edges and vertices. Using similar argument as in [2], it can be shown that each edge of two neighboring regions ${ }^{1} \mathcal{V}^{k}\left(H_{1}, S\right)$ and $\mathcal{V}^{k}\left(H_{2}, S\right)$ in $V^{k}(S)$ is the bisector of some pair of disks $\mathcal{C}_{i}$ and $\mathcal{C}_{j}$, where $\mathcal{C}_{i} \in H_{1}$ and $\mathcal{C}_{j} \in H_{2}$ More importantly, $H_{1}$ is different from $H_{2}$ by only one element. Since $\mathcal{C}_{i} \in H_{1}, \mathcal{C}_{j} \in H_{2}$, and $\mathcal{C}_{i} \neq \mathcal{C}_{j}$, this means that $H_{1}=H \bigcup\left\{\mathcal{C}_{i}\right\}$, and $H_{2}=H \bigcup\left\{\mathcal{C}_{j}\right\}$. Additionally, $d_{\max }\left(p, \mathcal{C}_{i}\right)=d_{\max }\left(p, \mathcal{C}_{j}\right) \geq d_{\max }\left(p, \mathcal{C}_{n}\right)$, $\forall \mathcal{C}_{n} \in H$. This is illustrated in Figure 2a.

The vertices in the order- $k$ max VD is classified as either new, or old. An vertex is old if it is the intersection of 3 max-Voronoi regions $\mathcal{V}^{k}\left(H_{1}, S\right), \mathcal{V}^{k}\left(H_{2}, S\right)$, and $\mathcal{V}^{k}\left(H_{3}, S\right)$, such that $H_{1}=H \bigcup\{a, b\}, H_{2}=H \bigcup\{b, c\}$, and $H_{3}=H \bigcup\{c, a\}$. On the other hand, a vertex is new if $H_{1}=H \bigcup\{a\}, H_{2}=H \bigcup\{b\}$, and $H_{3}=H \bigcup\{c\}$. For example, vertex $v_{1,6,7}$ (denoted by $v(C l, C 6, C 7)$ ) in Figure $2 \mathrm{a}$ is an old vertex. In this case, $H_{1}=\left\{\mathcal{C}_{1}, \mathcal{C}_{6}\right\}$, $H_{2}=\left\{\mathcal{C}_{6}, \mathcal{C}_{7}\right\}, H_{3}=\left\{\mathcal{C}_{7}, \mathcal{C}_{1}\right\}$, and $H=\emptyset$. Vertex $v_{4,5}$, is new since $H_{1}=\left\{\mathcal{C}_{6}, \mathcal{C}_{7}\right\}, H_{2}=\left\{\mathcal{C}_{6}, \mathcal{C}_{5}\right\}, H_{3}=\left\{\mathcal{C}_{6}, \mathcal{C}_{4}\right\}$, and $H=\left\{\mathcal{C}_{6}\right\}$.

The order- $k$ max-Voronoi region of an arbitrary set $H \subset S$ does not always exist. Therefore, efficient construction of highorder max-Voronoi diagram requires selecting the set of disks with a non-empty order- $k$ max-Voronoi region. We propose an incremental procedure to construct the order- $k$ max VD of a set of disks, which is based on the following lemma.

Lemma 2: Given a set of disks $S$, and $H=\left\{\mathcal{C}_{1}, \ldots, \mathcal{C}_{k}\right\} \subset$ $S, k>1$. Let $H_{i}=H-\left\{\mathcal{C}_{i}\right\}$, where $i=1, \ldots, k$, be the subsets of $H$ such that $\mathcal{V}^{k-1}\left(H_{i}, S\right) \neq \emptyset$. Then,

$$
\mathcal{V}^{k}(H, S) \equiv \bigcup_{i=1}^{k}\left(\mathcal{V}^{k-1}\left(H_{i}, S\right) \bigcap \mathcal{V}^{1}\left(\mathcal{C}_{i}, S-H_{i}\right)\right)
$$

Lemma 2 shows that the order- $k$ max VD can be constructed from the order- $(k-1)$ and the order-1 max VDs. Accordingly, each region $\mathcal{V}^{k-1}(H, S)$ of the order- $(k-1) \max$ VD is independently processed, in which the max VD of $(S-H)$, $V(S-H)$, is constructed. The set $H \bigcup\left\{\mathcal{C}_{j}\right\}$, such that $\mathcal{V}_{j}(S-H) \cap \mathcal{V}^{k-1}(H, S) \neq \emptyset$, must have an order- $k$ maxVoronoi region. The segments of edges of $\mathcal{V}_{j}(S-H)$ that are inside $\mathcal{V}^{k-1}(H, S)$ form the edges of $\mathcal{V}^{k}\left(H \bigcup\left\{\mathcal{C}_{i}\right\}, S\right)$.

When there is no trivial disk, it can be shown that only max-Voronoi regions of disks associated with new vertices of $\mathcal{V}^{k-1}(H, S)$ may overlap with $\mathcal{V}^{k-1}(H, S)$. This reduces the complexity in the incremental steps. An example is given in Figure 2, which shows the order-2 max VD of the set of disks $S=\left\{\mathcal{C}_{1}, \ldots, \mathcal{C}_{7}\right\}$. Figure $2 \mathrm{~b}$ illustrates the order- 1 max VD of 5 disks $\left\{\mathcal{C}_{1}, \ldots, \mathcal{C}_{5}\right\}$ that tessellates the order-2 max-Voronoi region $\mathcal{V}^{2}\left(\left\{\mathcal{C}_{6}, \mathcal{C}_{7}\right\}, S\right)$. As seen in the figures, $\mathcal{C}_{2}$ does not share a vertex with $\mathcal{C}_{6}$ or $\mathcal{C}_{7}$, thus its max-Voronoi region does not overlap $\mathcal{V}^{2}\left(\left\{\mathcal{C}_{6}, \mathcal{C}_{7}\right\}, S\right)$.

Let $H^{\prime}$ be the set of disks associated with the new vertices of $\mathcal{V}^{k-1}(H, S)$. Consider two disks $\mathcal{C}_{i}$ and $\mathcal{C}_{j}$ of $H^{\prime}$ with an

\footnotetext{
${ }^{1}$ Two max-VD regions of $V(S)$ are neighbors if they share at least an edge in $V(S)$.
} 


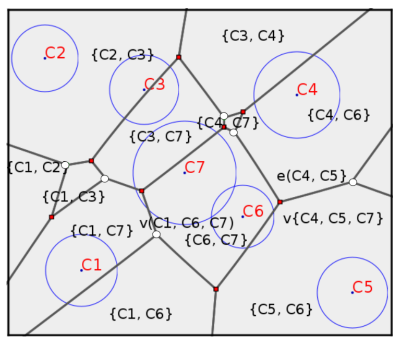

(a) order-2 $\max$ VD of $\left\{\mathcal{C}_{1}, \ldots, \mathcal{C}_{7}\right\}, \quad\{., \cdot\} \quad$ is the order- 1 max max-Voronoi region of two disks. Dashed arcs present the max VD of Solid arcs present the order- 2 max $\left\{\mathcal{C}_{1}, \ldots, \mathcal{C}_{5}\right\}$ VD.

Fig. 2: Extension of other disks' regions after removing some disks. The light dots present old vertices, the dark ones present new vertices.

edge $e_{i, j}$ incident at $v_{i, j, l}$ where $\mathcal{C}_{l} \in H$. By the previous argument, there exists a disk that is internally tangent to $\mathcal{C}_{i}$ and $\mathcal{C}_{j}$, and does not contain any other disk in its interior. This property clearly holds after deletion of some disks in $S$. Due to Lemma 1, there exists an edge $e_{i, j}^{\prime}$ in $V(S-H)$. Clearly, $v_{i, j, l} \in e_{i, j}^{\prime}$ in $V(S-H)$. In fact, $e_{i, j} \subset e_{i, j}^{\prime}$ with the latter expanding toward $\mathcal{V}^{k-1}(H, S)$. Moreover, it can be shown that the intersections of $V(S-H)$ and $\mathcal{V}^{k-1}(H, S)$ only occur at the new vertices of $\mathcal{V}^{k-1}(H, S)$.

Again, consider the example given in Figure 2. When $\mathcal{C}_{7}$ and $\mathcal{C}_{6}$ are removed, the max VD of $\left\{\mathcal{C}_{1}, \ldots, \mathcal{C}_{5}\right\}$ intersects with $\mathcal{V}^{2}\left(\left\{\mathcal{C}_{6}, \mathcal{C}_{7}\right\}, S\right)$. Consequently, the order-1 max-Voronoi regions of disks $\left\{\mathcal{C}_{1}, \mathcal{C}_{3}, \mathcal{C}_{4}, \mathcal{C}_{5}\right\}$ tessellate $\mathcal{V}^{2}\left(\left\{\mathcal{C}_{6}, \mathcal{C}_{7}\right\}, S\right)$ at its new vertices. For instance, the edge $e_{4,5}$ of $\mathcal{C}_{5}$ extends to $\mathcal{V}^{2}\left(\left\{\mathcal{C}_{6}, \mathcal{C}_{7}\right\}, S\right)$ at $v_{4,5,7}$. No tessellation happens at old vertices of $\mathcal{V}^{2}\left(\left\{\mathcal{C}_{6}, \mathcal{C}_{7}\right\}, S\right)$.

Algorithm 2 summarizes the construction of the order- $k$ $\max$ VD of $S$ from the order- $(k-1)$ VD with new and old vertices properly classified.

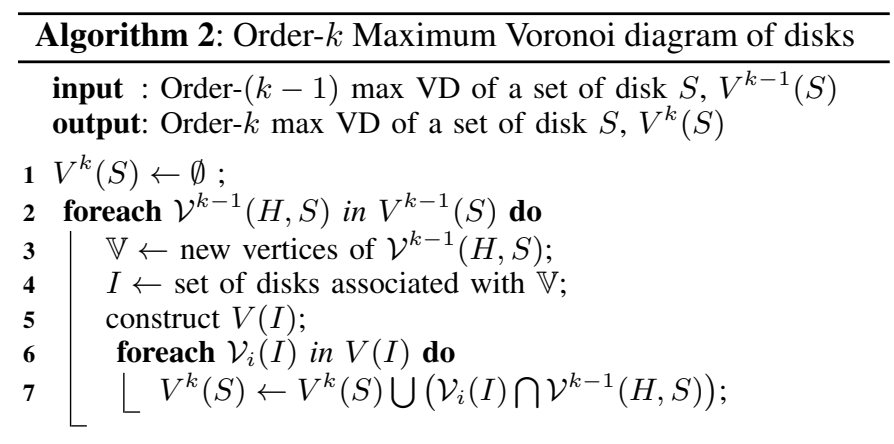

We derive the following results on the asymptotic behavior of the order- $k$ max VD:

Theorem 1: The order- $k \max \mathrm{VD}$ of $n$ disks can be constructed in $O\left(k^{2} \cdot T(n)\right)$, where $T(n)$ is the cost to construct the order-1 max VD.

Lemma 3: The average running time of $k$ nearest neighbors query among the set of $n$ disks, $N N Q(n, k)$, is $O(\log k n)$.
Note that, since $k \leq n$, the average running time of $k$ nearest neighbors query in Lemma 3 is in fact bounded by $O(2 \log n)$.

\section{ApPlication OF ORdER- $k$ MAX VD in RoBUST COVERAGE}

We apply the order- $k$ max VD to address the two problems raised in Section II, namely, guaranteed point $k$-coverage and area $k$-coverage. The objective is to determine the max-min sensing ranges in both problems.

\section{A. Guaranteed point k-coverage}

Given any point $p$ in the interested area and $V(S)$, we first determine the set $H$ of cardinality $k$, which are closer to $p$ than any disk in $S-H$. The minimum sensing range to ensure $k$-coverage to $p$ is, thus, given by,

$$
\gamma_{k}(p)=\max _{j \in H}\left\{\max _{q \in \mathcal{C}_{j}} d(p, q)\right\}=\max _{j \in H}\left\{d\left(o_{j}, p\right)+r_{j}\right\} .
$$

\section{B. Guaranteed area $k$-coverage}

Given a polygonal area $\boldsymbol{B}$ of interest, providing guaranteed $k$-coverage to $\boldsymbol{B}$ is equivalent to find a sensing range $\gamma_{k}$ ensuring $k$-coverage to any point $p \in \boldsymbol{B}$. We show that, it suffices to consider only the vertices of $V^{k}(S)$, the intersections of $V^{k}(S)$ and $\overline{\boldsymbol{B}}$, and the vertices of $\overline{\boldsymbol{B}}$. Accordingly, given the set of sensors $S$, the area $\boldsymbol{B}$ is tessellated into faces $\mathcal{F}(H)$ for $|H|=k$ and $\mathcal{V}^{k}(H, S) \neq \emptyset$. The boundary of $\mathcal{F}(H)$ consists of hyperbolic arcs due to order- $k$ max VD edges (or a subsegment), and line segments in $\overline{\boldsymbol{B}}$.

Lemma 4: Let $T$ and $U$ be the set of vertices of the boundary $\overline{\boldsymbol{B}}$, and intersections of $\overline{\boldsymbol{B}}$ and $V^{k}(S)$, respectively. The area $\overline{\boldsymbol{B}} \cup \boldsymbol{B}$ is $k$-covered iff all points in $T, U$, and Voronoi vertices of $V^{k}(S)$ are $k$-covered for sensing range $\gamma_{k}$.

\section{CONCLUSIONS AND FUTURE WORK}

We have devised geometrical-based methods to compute the minimum sensing range to ensure robust $k$ point and area coverage in presence of sensor location uncertainty. Our ongoing work extends the proposed scheme to build the order$k$ max VD directly from the order- $k$ VD of nominal points, which eliminates the constraint of trivial circles in this work. This will be applied to object tracking under uncertainty in wireless sensor network.

\section{REFERENCES}

[1] Franz Aurenhammer, Technische Universitt Graz, Rolf Klein, Fernuniversitt Hagen, and Praktische Informatik Vi. Voronoi diagrams. In Handbook of Computational Geometry, pages 201-290. Elsevier Science Publishers B.V. North-Holland.

[2] Der-Tsai Lee. On k-nearest neighbor voronoi diagrams in the plane. IEEE Trans. Comput., 31(6):478-487, 1982.

[3] X. Li, P. Wan, , and O. Frieder. Coverage in wireless ad hoc sensor networks. IEEE Transactions on Computers, 52(6), 2003.

[4] S. Meguerdichian, F. Koushanfar, M. Potkonjak, and M.B. Srivastava. Coverage problems in wireless ad-hoc sensor networks. In INFOCOM, 2001.

[5] H. Zhang and J. C. Hou. Maintaining sensor coverage in large scale sensor networks. In International Workshop on Theoretical and Algorithmic Aspects of Sensor, Ad hoc Wireless and Peer-to-Peer Networks, Feb. 2004. 